\title{
Dual-Energy CT in Hemorrhagic Progression of Cerebral Contusion: Overestimation of Hematoma Volumes on Standard 120-kV Images and Rectification with Virtual High- Energy Monochromatic Images after Contrast-Enhanced Whole-Body Imaging
}

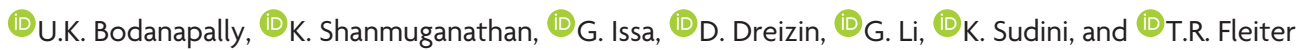

\begin{abstract}
BACKGROUND AND PURPOSE: In patients with hemorrhagic contusions, hematoma volumes are overestimated on follow-up standard 120-kV images obtained after contrast-enhanced whole-body CT. We aimed to retrospectively determine hemorrhagic progression of contusion rates on 120-kV and 190-keV images derived from dual-energy CT and the magnitude of hematoma volume overestimation.
\end{abstract}

MATERIALS AND METHODS: We retrospectively analyzed admission and follow-up CT studies in 40 patients with hemorrhagic contusions. After annotating the contusions, we measured volumes from admission and follow-up 120-kV and 190-keV images using semiautomated 3D segmentation. Bland-Altman analysis was used for hematoma volume comparison.

RESULTS: On 120-kV images, hemorrhagic progression of contusions was detected in 24 of the 40 patients, while only 17 patients had hemorrhagic progression of contusions on 190-keV images $(P=.008)$. Hematoma volumes were systematically overestimated on follow-up 120-kV images (9.68 versus $8 \mathrm{~mm}^{3}$; mean difference, $1.68 \mathrm{~mm}^{3}$; standard error, $0.37 ; P<.001$ ) compared with 190-keV images. There was no significant difference in volumes between admission 120-kV and 190-keV images. Mean and median percentages of overestimation were $29 \%(95 \% \mathrm{Cl}, 18-39)$ and $22 \%$ (quartile 3 - quartile $1=36.8$ ), respectively.

CONCLUSIONS: The 120-kV images, which are comparable with single-energy CT images, significantly overestimated the hematoma volumes, hence the rate of hemorrhagic progression of contusions, after contrast-enhanced whole-body CT. Hence, follow-up of hemorrhagic contusions should be performed on dual-energy CT, and 190-keV images should be used for the assessment of hematoma volumes.

ABBREVIATIONS: $\mathrm{DECT}=$ dual-energy $\mathrm{CT}$; $\mathrm{HPC}=$ hemorrhagic progression of contusion; $\mathrm{SECT}=$ single-energy $\mathrm{CT}$; $\mathrm{WBCT}=$ whole-body $\mathrm{CT}$

A pproximately 6 million patients with head and neck trauma are seen annually in the emergency departments of North America. ${ }^{1}$ One of the most severe types of traumatic brain injury is cerebral hemorrhagic contusion. ${ }^{2}$ Hemorrhagic contusions are usually complicated by secondary injury, resulting in hemorrhagic progression of contusion (HPC), which is designated as enlargement of the existing hemorrhagic contusions or appearance of new lesions. ${ }^{2}$ Several authors have reported rates of HPC in $38 \%-59 \%$ of cases. ${ }^{3-8}$ HPC is a progressive injury that results in irreversible loss of brain tissue with significant increase in morbidity

Received September 8, 2017; accepted after revision December 11.

From the Department of Diagnostic Radiology and Nuclear Medicine (U.K.B., K.S., G.I., D.D., G.L., T.R.F.), R Adams Cowley Shock Trauma Center, University of Maryland Medical Center, Baltimore, Maryland; and Department of Environmental Health Sciences (K.S.), Bloomberg School of Public Health, Johns Hopkins University, Baltimore, Maryland.

Please address correspondence to Uttam K. Bodanapally, MBBS, Department of Radiology, R Adams Cowley Shock Trauma Center, 22 S Greene St, University of Maryland Medical Center, Baltimore, MD 21201; e-mail: ubodanapally@umm.edu

http://dx.doi.org/10.3174/ajnr.A5558 and mortality. ${ }^{3}$ Noncontrast head CT is the diagnostic method used to assess patients with hemorrhagic contusions and HPC. ${ }^{3}$

There is increasing availability of dual-energy CT (DECT) technology in major academic and level 1 trauma centers. We have included a high-monochromatic (190-keV) image set in our routine head CT protocol at the University of Maryland Shock Trauma Center and Adult Emergency Department due to its potential to positively affect the display of cortical contusions and subdural hematomas by decreasing the beam-hardening artifacts from cranial bones. ${ }^{9-11}$ Mixed 120-kV images derived from dualenergy data mirror typical single-energy CT (SECT) images used in clinical practice. We have frequently observed higher hemorrhagic contusion volumes on follow-up $120-\mathrm{kV}$ compared with 190-keV images (Fig 1A, -B). This discrepancy was seen in patients after admission contrast-enhanced whole-body CT (WBCT) imaging, which has become a widely used technique for the work-up of the patient with blunt polytrauma. ${ }^{12}$

Studies have shown an increase in capillary endothelial permeability in contusions and surrounding parenchyma. ${ }^{2}$ We hypoth- 

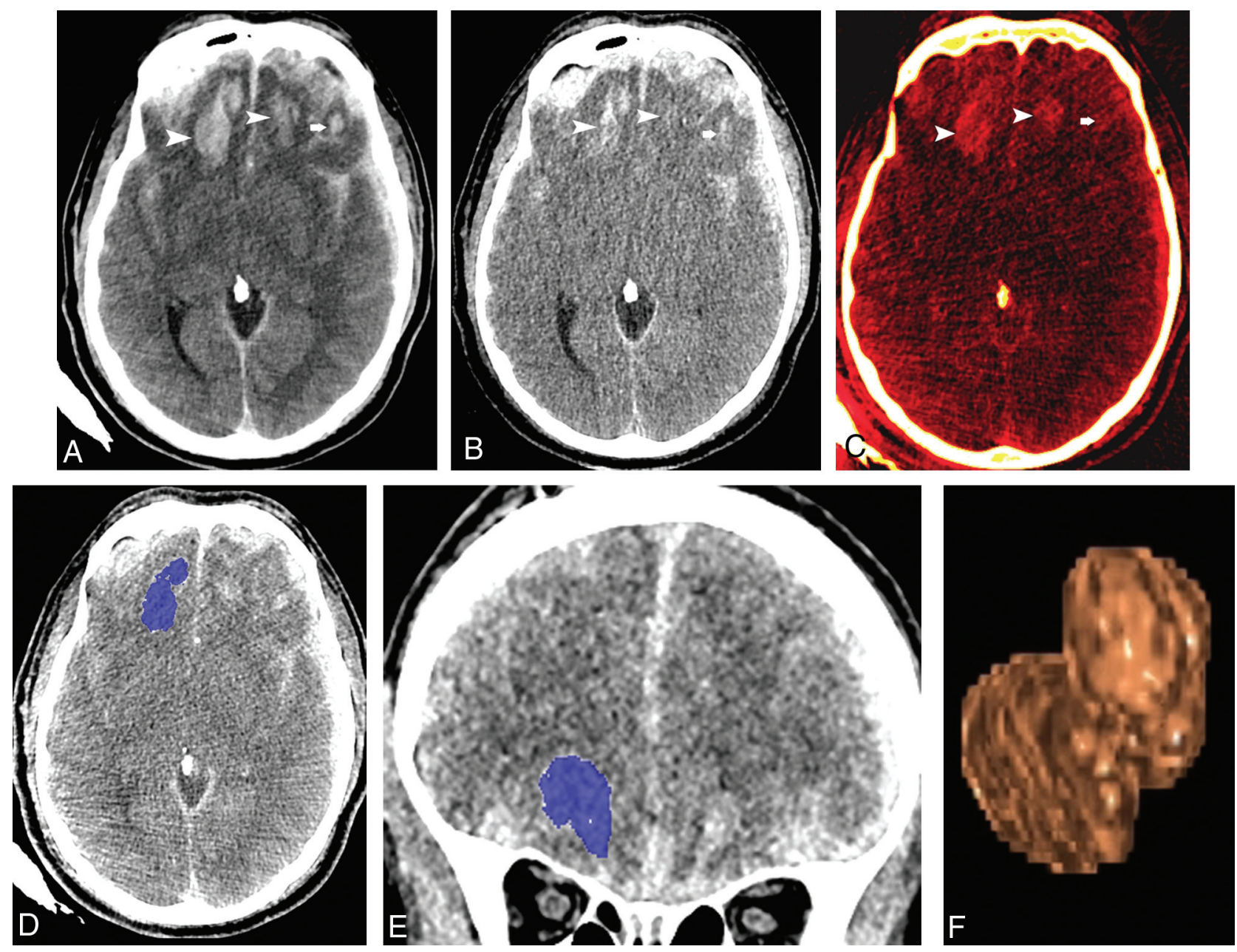

FIG 1. A 55-year-old man with traumatic brain injury sustained after an assault. A, Follow-up axial 120-kV image shows hemorrhagic contusions in both frontal lobes (arrowheads and arrow). B, Virtual high-monochromatic image (190-keV) shows significantly smaller hemorrhagic contusions compared with the $120-\mathrm{kV}$ images due to negligible attenuation contribution from leaked iodinated contrast (arrowheads and arrow). C, lodine overlay image shows contrast staining of the hemorrhagic contusions (arrowheads and arrow). Axial (D) and coronal (E) CT images demonstrate the ROIs drawn by 3D segmentation on the thin-client server. F, Segmented volume-rendered image of the hemorrhagic contusion.

esized that the discrepancy in measured volumes on follow-up noncontrast head CT is caused by retained iodinated contrast that leaks into the parenchyma through the permeable endothelium in the epicenter and penumbra of the contusions after admission contrast-enhanced WBCT (Fig 1C). The high attenuation of the leaked iodine resembles that of hematoma on $120-\mathrm{kV}$ images, resulting in volume overestimation. ${ }^{13-15}$ The linear attenuation of iodine shows a dramatic decrease along the spectrum of monochromatic energy, therefore greatly reducing the attenuation contribution of iodine at $190 \mathrm{keV}$, while at the same time, maintaining the attenuation of hematoma relatively constant. ${ }^{16,17}$ Hence, at clinically relevant tissue iodine concentrations, the attenuation contribution of iodine tends to be negligible at $190 \mathrm{keV}$ and demonstrates only the attenuation resulting from hematoma, thus allowing true measurement of hematoma volume. ${ }^{13,16,17}$ This phenomenon needs to be substantiated in a systematic study. We aimed to retrospectively determine HPC rates on $120-\mathrm{kV}$ and 190-keV images derived from DECT data and the magnitude of hematoma volume overestimation on follow-up 120-kV images in patients after admission contrast-enhanced WBCT.

\section{MATERIALS AND METHODS}

This retrospective study was Health Insurance Portability and Accountability Act-compliant, and permission was obtained from University of Maryland, Baltimore institutional review board. Informed consent was waived. Consecutive series of patients referred to the trauma resuscitation unit were eligible. The inclusion criteria were the following: 1) a history of blunt trauma with acquisition of noncontrast head DECT followed by contrast-enhanced WBCT, as a part of diagnostic work-up of blunt polytrauma, between May 15, 2016, and September 10, 2016, with a confirmed diagnosis of hemorrhagic contusion on admission CT; 2) acquisition of follow-up noncontrast head DECT within 3 days of admission CT; and 3) 18 years of age or older. Patients were excluded if the mechanism of injury was penetrating trauma or if they underwent surgical interventions to address their contusions.

\section{Subjects}

A search of our radiology information system data base from the designated period yielded 171 patients with initial and at least 1 follow-up study performed on a DECT scanner. A radiologist 
with 11 years of experience reviewed the initial and follow-up DECT studies to select all the patients with hemorrhagic contusions $(n=40)$ that constituted the study group. The mean age of the final cohort was 38.4 years (range, 18-73 years), with 26 men and 14 women.

\section{Reference Standard}

HPC is defined as an enlargement of $\geq 30 \%$ of the original hematoma volume on follow-up CT studies as suggested by Alahmadi et al. ${ }^{18}$

\section{Imaging Technique}

Admission WBCT and follow-up head examinations were performed on a DECT scanner (Somatom Force; Siemens, Erlangen, Germany). WBCT involves a noncontrast head CT followed by contrast-enhanced CT of the neck, chest, abdomen, and pelvis. The studies were performed after injection of $100 \mathrm{~mL}$ of iodinated contrast media (iohexol, Omnipaque 300; GE Healthcare, Piscataway, New Jersey) using a biphasic injection with $60 \mathrm{~mL}$ injected at $5 \mathrm{~mL} / \mathrm{s}$ and $40 \mathrm{~mL}$ injected at $4 \mathrm{~mL} / \mathrm{s}$. The contrast injection was followed by a $50-\mathrm{mL}$ saline injection at $4 \mathrm{~mL} / \mathrm{s}$.

DECT head images were obtained with the $\mathrm{x}$-ray tubes at $80 \mathrm{kV}$ and $\mathrm{Sn} 150 \mathrm{kV}(150 \mathrm{kV}+$ tin filter). Scan parameters were as follows: rotation time, 0.5 seconds; pitch, 0.55 . The reference milliampere-second was 273 for the Sn150kV and 410 for the $80-\mathrm{kV}$ tube. Original dual-energy datasets were reconstructed with an increment of $1 \mathrm{~mm}$ and a slice thickness of $1 \mathrm{~mm}$. Automatic reconstruction of $120-\mathrm{kV}$-equivalent mixed-DECT images at 5-mm slice thickness and 5-mm intervals using an adaptive iterative reconstruction algorithm (ADMIRE, Siemens) with a strength value of 3 was performed and sent to the PACS at the time of the study. Automatic tube current modulation (CARE Dose4D; Siemens) was used in all patients.

\section{Image Analysis of DECT}

DECT data from admission and follow-up head CTs were processed to derive 190-keV image sets at 5-mm slice thickness and 5-mm intervals on a workstation (syngo.via, Version VB10B; Siemens) and sent to the PACS. Both $120-\mathrm{kV}$ and 190-keV image sets from the PACS (5-mm slice thickness, 5-mm intervals) were loaded onto the thin-client server at our institution (IntelliSpace Portal; Philips Healthcare, Best, the Netherlands) to facilitate hematoma volume measurements. Hence, 4 image sets were used for volume measurements in each patient (ie, $120-\mathrm{kV}, 190-\mathrm{keV}$ image sets [from the admission study] and $120-\mathrm{kV}, 190-\mathrm{keV}$ [from the follow-up study]). Reviewer 1 annotated the hemorrhagic contusions that were meant for volume measurements. In patients with multiple contusions, the largest lesion was used for measurement. Reviewer 2 (third-year radiology resident) measured the volumes of the hemorrhagic contusions in each patient. Measurements were randomly performed regarding patient order and the order of the image sets to avoid potential preconceived bias. Volumes were measured using semiautomated 3D segmentation. An ROI was drawn on axial slices with the use of coronal and sagittal slices to exclude unwanted surrounding brain tissue from the ROIs (Fig 1D-F). ${ }^{19}$

\section{Statistical Analysis}

Statistical analysis was performed by K.S. using statistical software (JMP 12 software; SAS Institute, Cary, North Carolina). Contingency analysis was used to compare dichotomous variables. The $\mathrm{McNemar}$ test was used to compare the frequencies of HPC on $120-\mathrm{kV}$ and 190-keV images. For comparison of hematoma volumes obtained by $120-\mathrm{kV}$ and $190-\mathrm{keV}$, Bland-Altman analysis was performed. To determine the correlation of volume measurements on both image sets, we calculated the Pearson correlation coefficient. The Wilcoxon signed rank test was used to test the potential differences between the median values obtained by both methods. Regression analysis was performed to identify the best predictor of the percentage of hematoma volume overestimation. The following formulas were used to calculate the different variables: Percentage of Hematoma Overestimation $=100$ (Volume on Follow-Up 120-kV - Volume on Follow-Up 190-keV) / (Volume on Follow-Up 190-keV); Percentage of Hemorrhagic Progression on $190 \mathrm{keV}=100$ (Volume of Hematoma on Follow-Up 190-keV - Volume on Admission 190-keV) / (Volume on Admission 190-keV); similarly, the percentage of hemorrhagic progression on $120 \mathrm{kV}=100$ (Volume of Hematoma on Follow-Up $120-\mathrm{kV}$ - Volume on Admission 120-kV) / (Volume on Admission $120-\mathrm{kV})$. A $P$ value of $<.05$ was considered significant.

\section{RESULTS}

The median time to follow-up noncontrast head CT was 6 hours (quartile 3 - quartile $1=4.75$ hours) after admission contrastenhanced WBCT. On follow-up 120-kV images, HPC was detected in 24 of the 40 patients $(60 \%)$, with HPC defined as an enlargement of $\geq 30 \%$ of the original hematoma volume. On 190$\mathrm{keV}$ images, HPC was detected in only 17 of the 40 patients (43\%). The McNemar test showed that HPC was more frequently observed on $120-\mathrm{kV}$ images with a test result of $7(P=.008)$.

Bland-Altman analysis showed that hematoma volumes were systematically overestimated on follow-up $120-\mathrm{kV}$ images ( 9.68 versus $8 \mathrm{~mm}^{3}$; mean difference, $1.68 \mathrm{~mm}^{3}$; standard error, 0.37 ; $P<.001$ ) compared with $190-\mathrm{keV}$ images (Fig 2). There was no significant difference in the measured volumes between admission $120-\mathrm{kV}$ and $190-\mathrm{keV}$ images (6.11 versus $6.07 \mathrm{~mm}^{3}$; mean difference, $0.045 \mathrm{~mm}^{3}$; standard error, $\left.0.05 ; P=.22\right)$. The Pearson correlation coefficient for hematoma volumes determined by $120-\mathrm{kV}$ and $190-\mathrm{keV}$ was $r=0.999(P<.001)$ for admission head CTs and $r=0.98(P<.001)$ for follow-up head CTs. The mean percentage of hematoma volume overestimation on follow-up $120-\mathrm{kV}$ images was $29 \% \pm 32 \%$ (95\% CI, $18 \%-39 \%)$, and the median was $22 \%$ (quartile 3 - quartile $1=36.8$ ).

Regression analysis was performed using the hematoma volume on admission CT (190-keV), hematoma volume on follow-up CT (190-keV), time to follow-up, absolute volume of hemorrhagic progression (190-keV), and percentage of hemorrhagic progression as independent variables with percentage of hematoma overestimation as a dependent variable. The percentage of HPC was the best predictor of the percentage of hematoma volume overestimation ( $\beta=16.4$; 95\% CI, 9.91-22.97; standard error, $3.2 ; P<.001)$. 


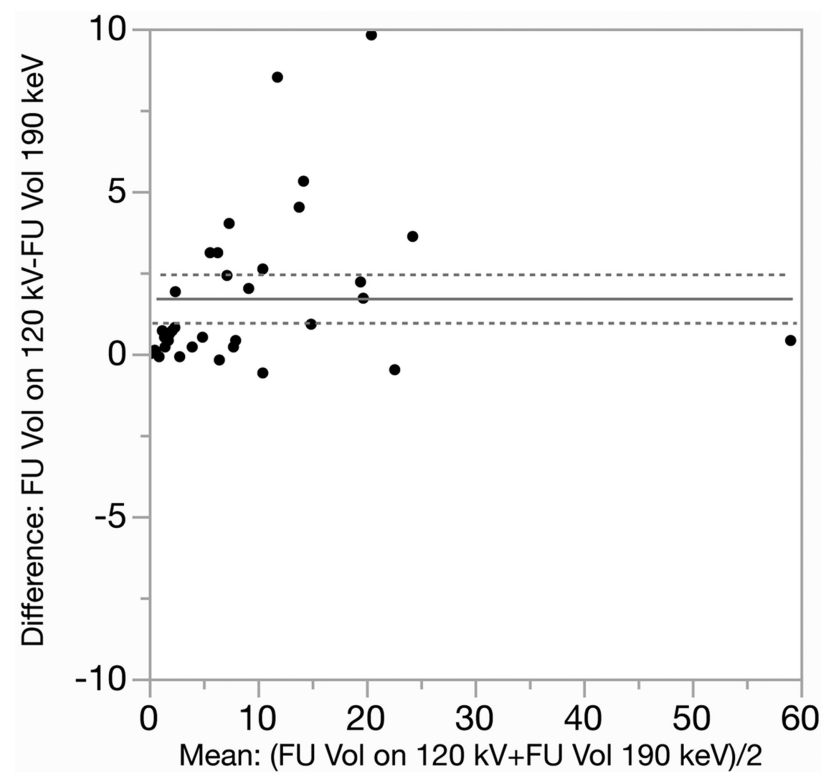

FIG 2. Bland-Altman plots of the hematoma volume overestimation on follow-up $120-k V$ images compared with $190-k e V$ images. Horizontal lines represent the mean volume overestimation (solid line) and limits of agreement (dashed lines). FU indicates follow-up; Vol, volume.

\section{DISCUSSION}

The major findings from our study in patients after admission contrast-enhanced WBCT are the following: 1) follow-up 120-kV head CT images, which are akin to SECT images, significantly overestimate the hematoma volume and hence the rate of HPC, 2) mean and median percentages of hematoma volume overestimations were $29 \%$ and $22 \%$, respectively, and 3 ) the best predictor of percentage of volume overestimation was the percentage of hemorrhagic progression on follow-up CT (ie, the magnitude of overestimation is proportional to the magnitude of HPC).

Our study demonstrated that the true occurrence of HPC in patients after contrast-enhanced WBCT, as measured from the $190-\mathrm{keV}$ images, was $43 \%$. This number is similar to the $45 \%$ that was reported in conservatively managed patients by Alahmadi et al, ${ }^{18}$ who had similar selection criteria and cutoff values for progression. However, the rate increased to $60 \%$ if measured on $120-\mathrm{kV}$ images (images comparable with SECT images) because there were 7 patients (18\%) who were wrongly diagnosed with HPC. This higher rate of HPC is caused by "pseudohematoma." We describe pseudohematoma as the volume of nonhemorrhagic hyperattenuating brain parenchyma caused by iodinated contrast leak from the permeable capillary endothelium. The leaked contrast from the bolus administered during the admission WBCT is retained in the brain parenchyma and would be seen in the follow-up studies as an area of hyperattenuation on $120-\mathrm{kV}$ images, simulating a hematoma. The exact duration of this parenchymal contrast retention has not yet been analyzed and reported.

The attenuation contribution of iodine is negligible at the higher end of the monochromatic energy spectrum. ${ }^{13,16,17}$ Hence, $190-\mathrm{keV}$ images demonstrate the actual hematoma size by displaying attenuation contributed predominantly by hematoma. It was recently demonstrated that virtual noncontrast images are comparable with $190-\mathrm{keV}$ images in minimizing the attenuation contribution of iodine to negligible levels. ${ }^{13}$ Hence, virtual noncontrast images, if used in place of $190-\mathrm{keV}$ images, may have yielded similar results in obtaining true hematoma volumes. Enhancement of cerebral contusion on contrast-enhanced SECT was described by Huang et $\mathrm{al}^{20}$; however, the authors did not describe how they were able to differentiate the hyperattenuation caused by iodinated contrast from that of the hematoma.

Cerebral contusions can be divided into 3 distinct regions: 1 ) epicenter, 2) penumbra, and 3) parapenumbra. ${ }^{2}$ The epicenter receives the peak energy from impact, and surrounding regions receive progressively less energy with distance. Energy deposited in the epicenter is sufficient to fracture capillaries, resulting in an immediate hemorrhagic lesion. In the penumbra and parapenumbra, the energy is not enough to fracture the capillaries but activates the mechanosensitive molecular processes that will lead to the delayed structural failure of capillaries. ${ }^{21,22}$ Animal studies have shown that the mechanosensitive molecular process begins with transcriptional up-regulation, followed by opening of the sulfonylurea receptor 1 (SUR1) SUR1-regulated channel. ${ }^{2,21}$ Opening the channel has been linked to gradual oncotic cell swelling and death of the endothelial cells, resulting in increased capillary permeability, and progression to capillary fragmentation. Increased permeability results in vasogenic edema, while capillary fragmentation results in extravasation of blood, contributing to HPC. HPC rates are directly related to the degree of endothelial damage and capillary fragmentation. The penumbra and parapenumbra usually manifest as vasogenic edema on CT because of increased permeability of the damaged endothelium. ${ }^{2}$ However, after contrast administration, enhancement of the parenchyma in the penumbra and parapenumbra results from the leak of iodinated agents through the same damaged endothelium, resulting in pseudohematoma. ${ }^{20}$ The phenomenon of HPC rates being proportional to the degree of capillary disruption caused by the mechanosensitive molecular process likely explains our other finding (ie, the magnitude of overestimation of hematoma is proportional to the magnitude of HPC because both are directly related to the degree of endothelial damage and capillary fragmentation).

Several authors have reported HPC as the reason for an operation, sometimes in up to $20 \%$ of patients. ${ }^{3-8}$ Studies have also reported that patients with progression of injury on a repeat CT underwent more frequent interventions related to intracranial pressure management changes, ventriculostomy placement or adjustment, addition of antiseizure medication, repeat CT, intensive care unit observation, delay in extubation, and initiation of thromboprophylaxis, even in patients with stable or improved Glasgow Coma Scale scores. ${ }^{12,18,23-25}$ Similarly, Alahmadi et al ${ }^{18}$ reported a significant association between radiographic progression of contusions and the need for neurosurgical interventions, even though the mean Glasgow Coma Scale score and mean change in Glasgow Coma Scale score during the hospital course showed no difference. ${ }^{18}$ Hence, an accurate assessment of HPC has important clinical implications in preventing unnecessary interventions and radiation from follow-up imaging.

Using strict criteria in describing HPC, including indicating whether there was prior contrast administration, and detailing image sets used for measurement (SECT, $120 \mathrm{kV}$, or $190 \mathrm{keV}$ ) 
would minimize potential errors in identifying contusion progression. Health care providers should account for overestimation of hematoma volumes if the follow-up studies were performed on SECT. We also suggest that future research articles describe in detail the imaging techniques in their methods section, because contrast-enhanced WBCT has become a widely used technique for the work-up of the patient with blunt polytrauma.

\section{Limitations}

Our study has several limitations. It is a retrospective single-center design, which introduces selection and institutional bias. The cohort comprised only those patients who underwent WBCT studies at the time of admission. Thus, these data may not be generalizable to all trauma patients who may have undergone focused imaging of the head. A lack of consistent protocol for follow-up CTs (though most scans were obtained within 9 hours) may have affected the values. Finally, we excluded patients who underwent neurosurgical interventions. Those patients may differ in the severity of endothelial damage and hence have a different magnitude of iodine leak and hematoma volume overestimation.

\section{CONCLUSIONS}

Mixed $120-\mathrm{kV}$ images from DECT and by extension the SECT images overestimate hematoma volumes, hence the HPC rates on follow-up head CTs, in patients after contrast-enhanced WBCT. Our study demonstrates that it is important to perform follow-up of cerebral contusions on a DECT scanner and use high-monochromatic (190-keV) images in patients after contrast-enhanced WBCT to accurately estimate HPC.

Disclosures: Uttam K. Bodanapally_UNRELATED: Payment for Lectures Including Service on Speakers Bureaus: Siemens, Comments: talk on the role of "Dual-Energy CT in Patients with Traumatic Brain Injury" at the American Society of Head and Neck Radiology Annual Meeting in Las Vegas, September 17, 2017; Travel/Accommodations/Meeting Expenses Unrelated to Activities Listed: Siemens, Comments: talk on the role of "Dual-Energy CT in Patients with Traumatic Brain Injury" at American Society of Head and Neck Radiology Annual Meeting in Las Vegas, September 17, 2017. David Dreizin—UNRELATED: Grants/Grants Pending: Radiological Society of North American research scholar grant, Siemens. * Money paid to the institution.

\section{REFERENCES}

1. Stiell IG, Clement CM, Grimshaw JM, et al. A prospective cluster-randomized trial to implement the Canadian CT Head Rule in emergency departments. CMAJ 2010;182:1527-32 CrossRef Medline

2. Kurland D, Hong C, Aarabi B, et al. Hemorrhagic progression of a contusion after traumatic brain injury: a review. $J$ Neurotrauma 2012;29:19-31 CrossRef Medline

3. Bodanapally UK, Sours C, Zhuo J, et al. Imaging of traumatic brain injury. Radiol Clin North Am 2015;53695-715 CrossRef Medline

4. Chang EF, Meeker M, Holland MC. Acute traumatic intraparenchymal hemorrhage: risk factors for progression in the early postinjury period. Neurosurgery 2006;58:647-56; discussion 647-56 CrossRef Medline

5. Lobato R, Gomez P, Alday R, et al. Sequential computerized tomography changes and related final outcome in severe head injury patients. Acta Neurochir 1997;139:385-91 CrossRef Medline

6. Servadei F, Nanni A, Nasi MT, et al. Evolving brain lesions in the first 12 hours after head injury analysis of 37 comatose patients. Neurosurgery 1995;37:899-904 CrossRef Medline
7. Miller JD, Butterworth JF, Gudeman SK, et al. Further experience in the management of severe head injury. J Neurosurg 1981;54:289-99 CrossRef Medline

8. Cepeda S, Gómez PA, Castaño-Leon AM, et al. Traumatic intracerebral hemorrhage: risk factors associated with progression. $J \mathrm{Neu}$ rotrauma 2015;32:1246-53 CrossRef Medline

9. Yu L, Leng S, McCollough CH. Dual-energy CT-based monochromatic imaging. Am J Roentgenol 2012;199(5 Suppl):S9-15 CrossRef Medline

10. Wu X, Langan DA, Xu D, et al. Monochromatic CT image representation via fast switching dual kVp. Proc SPIE 2009;7258:725845

11. Matsumoto K, Jinzaki M, Tanami $Y$, et al. Virtual monochromatic spectral imaging with fast kilovoltage switching: improved image quality as compared with that obtained with conventional $120-\mathrm{kVp}$ CT. Radiology 2011;259:257-62 CrossRef Medline

12. Gunn ML, Kool DR, Lehnert BE. Improving outcomes in the patient with polytrauma: a review of the role of whole-body computed tomography. Radiol Clin North Am 2015;53:639-56 CrossRef Medline

13. Bodanapally UK, Dreizin D, Issa G, et al. Dual-energy CT in enhancing subdural effusions that masquerade as subdural hematomas: diagnosis with virtual high-monochromatic (190-keV) images. AJNR Am J Neuroradiol 2017;38:1946-52 CrossRef Medline

14. Gupta R, Phan CM, Leidecker C, et al. Evaluation of dual-energy CT for differentiating intracerebral hemorrhage from iodinated contrast material staining. Radiology 2010;257:205-11 CrossRef Medline

15. Phan CM, Yoo AJ, Hirsch JA, et al. Differentiation of hemorrhage from iodinated contrast in different intracranial compartments using dual-energy head CT. AJNR Am J Neuroradiol 2012;33:1088-94 CrossRef Medline

16. Postma AA, Das M, Stadler AA, et al. Dual-energy CT: what the neuroradiologist should know. Curr Radiol Rep 2015;3:16 CrossRef Medline

17. Potter CA, Sodickson AD. Dual-energy CT in emergency neuroimaging: added value and novel applications. Radiographics 2016;36: 2186-98 CrossRef Medline

18. Alahmadi H, Vachhrajani S, Cusimano MD. The natural history of brain contusion: an analysis of radiological and clinical progression. J Neurosurg 2010;112:1139-45 CrossRef Medline

19. Bodanapally UK, Addis H, Dreizin D, et al. Prognostic predictors of visual outcome in open globe injury: emphasis on facial CT findings. AJNR Am J Neuroradiol 2017;38:1013-18 CrossRef Medline

20. Huang AP, Lee CW, Hsieh HJ, et al. Early parenchymal contrast extravasation predicts subsequent hemorrhage progression, clinical deterioration, and need for surgery in patients with traumatic cerebral contusion. J Trauma 2011;71:1593-99 CrossRef Medline

21. Simard JM, Kilbourne M, Tsymbalyuk O, et al. Key role of sulfonylurea receptor 1 in progressive secondary hemorrhage after brain contusion. J Neurotrauma 2009;26:2257-67 CrossRef Medline

22. Patel AD, Gerzanich V, Geng Z, et al. Glibenclamide reduces hippocampal injury and preserves rapid spatial learning in a model of traumatic brain injury. J Neuropathol Exp Neurol 2010;69:1177-90 CrossRef Medline

23. Wang MC, Linnau KF, Tirschwell DL, et al. Utility of repeat head computed tomography after blunt head trauma: a systematic review. J Trauma 2006;61:226-33 CrossRef Medline

24. Lee TT, Aldana P, Kirton O, et al. Follow-up computerized tomography $(\mathrm{CT})$ scans in moderate and severe head injuries: correlation with Glasgow Coma Scores (GCS), and complication rate. Acta Neurochir 1997;139:1042-47; discussion 1047-48 CrossRef Medline

25. Givner A, Gurney J, O'Connor D, et al. Reimaging in pediatric neurotrauma: factors associated with progression of intracranial injury. J Pediatr Surg 2002;37:381-85 CrossRef Medline 